\title{
Overview of Multiquark States
}

\author{
Christian S. Fischer* \\ Institut für Theoretische Physik, Justus-Liebig-Universität Giessen, 35392 Giessen, Germany \\ E-mail: christian.fischerephysik.uni-giessen.de
}

\section{Gernot Eichmann}

CFTP, Instituto Superior Técnico, Universidade de Lisboa, 1049-001 Lisboa, Portugal

E-mail: gernot.eichmann@tecnico.ulisboa.pt

In this contribution we focus on the spectrum of three-quark (qqq) and four-quark $(\bar{q} q \bar{q} q)$ states in the framework of Dyson-Schwinger, Bethe-Salpeter, Faddeev and Faddeev-Yakubowsky equations. To determine both types of spectra we use a well-established momentum-dependent quarkgluon interaction that generates dynamical chiral symmetry breaking in accordance with chiral identities. We discuss recent results on the spectrum of ground-state and excited baryons in the light and strange quark sector. For double and triple strange baryons we provide predictions which can be explored in current and future experiments such as LHCb and PANDA/FAIR. For the four-quark systems we exemplify the framework's potential to describe the transition between resonances and bound states with increasing quark masses and discuss the possibility to distinguish between various internal clusterings of heavy-light four-quark systems.

XVII International Conference on Hadron Spectroscopy and Structure - Hadron2017

25-29 September, 2017

University of Salamanca, Salamanca, Spain

\footnotetext{
${ }^{*}$ Speaker.
} 


\section{Introduction}

In this contribution to the proceedings of Hadron2017 we summarise recent progress on the properties of baryons and tetraquarks as obtained from the functional approach (Dyson-Schwinger, Bethe-Salpeter, Faddeev and Faddeev-Yakubowsky equations) to QCD. Some of the topics (spectrum of light baryons) are also covered in much more detail in a recent review [1].

One of the key elements in unravelling the structure of the strong interaction is the understanding of the baryon excitation spectrum of QCD extracted from experiments using electromagnetic and other probes. Significant experimental progress has been made in the past years by the analysis of data from photo- and electroproduction experiments at JLAB, ELSA and MAMI [2, 3, 4] resulting in a substantial number of additions to the PDG [5]. In the same time many interesting candidates for unconventional ("exotic") hadron states have been identified which do not seem to fit the conventional pattern of $q \bar{q}$ and $q q q$ states. Some of these are potential candidates for four-quark states with colour-neutral $q \bar{q} q \bar{q}$ content, see e.g. $[6,7,8]$ for reviews.

A number of non-perturbative methods contribute interesting aspects to our theoretical understanding of hadrons. While the quark model still serves as a kind of standard by which one may define and distinguish the 'expected' from the 'unexpected' [9, 10], its ignorance of many aspects of the dynamics of underlying QCD calls for more refined methods. For ground state masses, lattice QCD contributes its share with small error bars and very good agreement between different approaches. For excited states, however, a number of technical subtleties still prevent the emergence of a clear picture.

In the past years functional methods to treat non-perturbative QCD using Dyson-Schwinger, Bethe-Salpeter, Faddeev and Faddeev-Yakubowsky equations (DSEs, BSEs FEs, FYEs) have been extensively explored. In contrast to many model approaches, this framework can be used to deal with mesons, baryons and exotic states in the light and heavy quark sector using the same underlying approximation for the quark-gluon interaction. This unified treatment offers the possibility to systematically explore and compare the effects of different elements of the QCD dynamics on hadron spectra, structure and dynamics [1]. While we discuss corresponding results for the spectra of three- and four-body states in this contribution, an overview of corresponding results for form factors can be found in [1].

Our results for the octet and decuplet baryon spectrum will be discussed in Sec. 3. Results for light scalar four-body bound states and resonances will be the topic of Sec. 4 together with an outlook into the heavy quark sector. First, however, we outline the framework in the next section.

\section{The functional approach to baryons}

The masses and Faddeev amplitudes of baryons are extracted from their Faddeev equation, shown in Fig. 1 (the Faddeev amplitudes are illustrated by the shaded half-spheres). Its ingredients are the fully dressed quark propagator (solid line with open circle) as well as the quark two-body and irreducible three-body interactions. Besides their colour and flavour parts, the fully relativistic Faddeev amplitudes also contain a substantial number of Dirac tensor structures (64 for $J=\frac{1}{2}$ and 128 for $J=\frac{3}{2}$ baryons) with dressing functions dependent on the total and relative momenta of the quarks inside the baryons. 


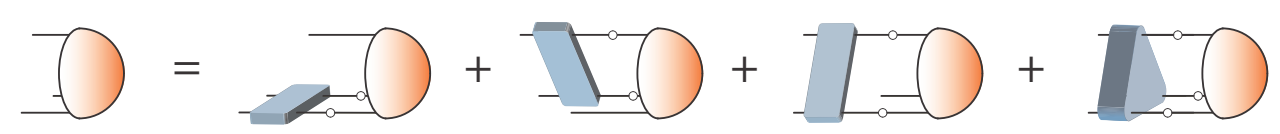

Figure 1: Three-quark Faddeev equation.

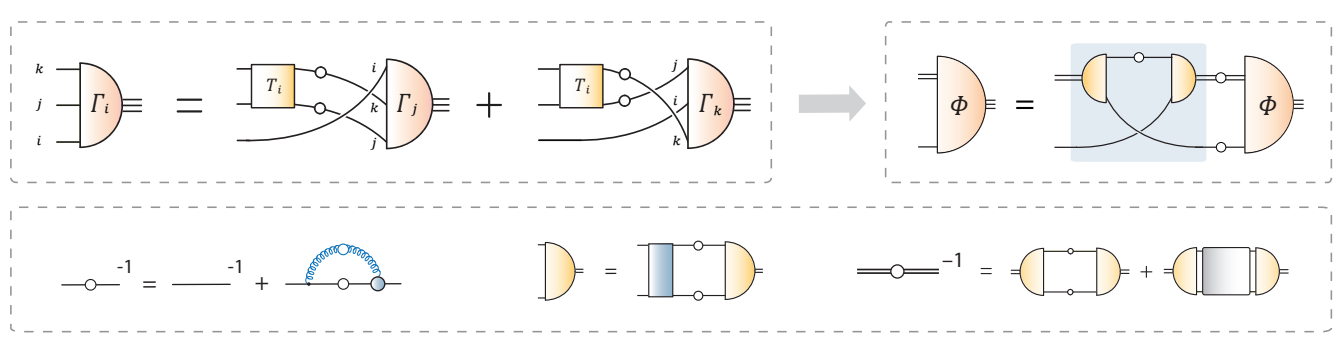

Figure 2: Simplification of the Faddeev equation in Fig. 1 to the quark-diquark Bethe-Salpeter equation (upper panel). The lower panel shows the ingredients that enter in the equation and are calculated beforehand: the quark propagator, diquark Bethe-Salpeter amplitudes and diquark propagators.

While the treatment of the three-body Faddeev equation has become well feasible in recent years, it is instructive to compare its results with those in a quark-diquark approximation, shown in the upper panel of Fig. 2, which simplifies matters considerably. Its main ingredients are again the dressed quark propagator, together with the diquark propagator (double line with open circle) as well as the diquark Bethe-Salpeter amplitude. In turn, the latter needs to be calculated from the diquark Bethe-Salpeter equation (bottom center diagram of Fig. 2). The dressed quark propagator is determined from its Dyson-Schwinger equation (bottom left diagram of Fig. 2).

An important question regarding the diquark approximation is how many diquarks to consider. While in principle the diquark expansion of the four-quark correlator includes ground and excited states of diquarks with any quantum numbers, in practice the two lightest types of diquarks, the scalar and axialvector ones [11], constitute a minimal set sufficient to build $J^{P}=1 / 2^{+}$octet and $J^{P}=3 / 2^{+}$decuplet baryons. For the remaining states, contributions from pseudoscalar and vector diquarks have to be taken into account [12] and are included in the results discussed below.

The three-body and quark-diquark approximation can only be systematically compared when the same underlying quark-gluon interaction is chosen. This is not possible in quark-diquark models that utilize ansaetze for the quark propagator and the diquark wave functions without contact to the underlying QCD dynamics $[13,14,15]$. Below we discuss results obtained from a rainbowladder type of interaction introduced in Ref. [16] and since used in many applications; details are discussed e.g. in $[17,1]$. Here the full interaction between two quarks is approximated by the exchange of an effective dressed gluon. The two parameters of this interaction, a scale and a shape parameter, are fixed in the meson sector: the scale is set by the pion decay constant, whereas the shape parameter can be varied in a range of values with only small changes in most experimental observables, see e.g. [18]. In addition, a third parameter adapted to the $\rho-a_{1}$ splitting is introduced to mimic QCD's different interaction strength in parity partner channels [19, 17]. The quark masses are adapted such that the masses of pseudoscalar mesons are reproduced. Thus all aspects of the quark-gluon interaction are completely fixed in the meson sector and the results for the spectra of baryons (and tetraquarks) are predictions. 

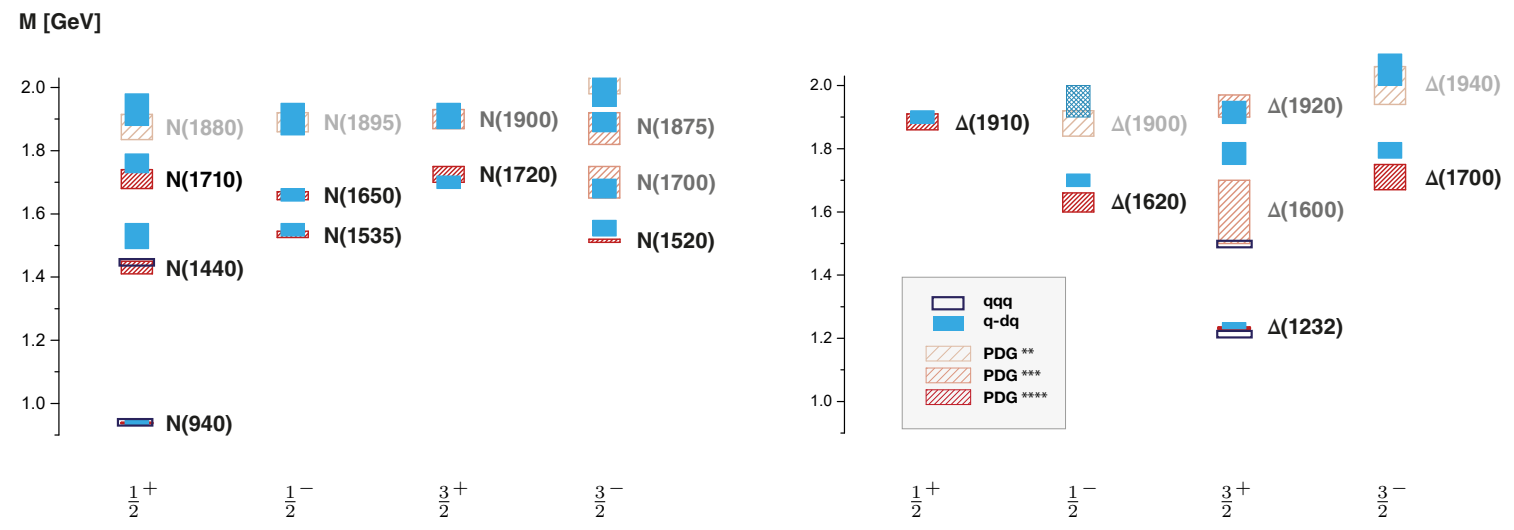

Figure 3: Nucleon and $\Delta$ baryon spectrum for $J^{P}=1 / 2^{ \pm}$and $3 / 2^{ \pm}$states determined within rainbow-ladder $[17,20]$. The three-body results (open boxes) are compared to the quark-diquark spectrum with full diquark content (filled boxes), together with the PDG values and their experimental uncertainties [5]. The widths of our results represent an estimate of the systematic error based on the extrapolated eigenvalue curves of the Faddeev equations, see [17] for details.

\section{Light and strange baryon spectrum}

In Fig. 3 we show the resulting spectra from the three-body calculation (open boxes) [21, 22], the quark-diquark approximation (filled boxes) [17] and compare them with the two-, three- and four-star states given by the PDG [5].

Let us first discuss the nucleon channel. In the left diagram we observe good agreement of the three-body with the quark-diquark approach for the ground-state nucleon as well as the first radially excited state. The mass of the latter agrees well with the Breit-Wigner mass of the Roper shown as a red shaded box ${ }^{1}$. The next two excited states are (numerically) only accessible in the quark-diquark framework and lie in the same ballpark as the PDG's N(1710) and N(1880). In the nucleon channel with negative parity the ( $\rho-a_{1}$ adjusted) quark-diquark calculation shows again ground and excited states in agreement with experiment. In particular the $\mathrm{N}(1535)$ is where it should be and the experimental level ordering as compared to the positive parity channel is correctly reproduced. Note that although the inclusion of the 'bad' diquarks of type pseudoscalar and vector is quantitatively important to reproduce the spectrum, the states in the negative parity channel are still dominated by the 'good' diquarks of scalar and axialvector type due to their lower masses. Thus, in agreement with the quark model and a recent quark-diquark model calculation [23], the negative parity states are dominated by contributions with one unit of angular momentum ( $p$ waves), although other contributions (s-wave and d-wave) are also present. In contrast, in the positive-parity channel the ground-state nucleon is s-wave dominated, whereas the first excited state, the Roper, is dominated by p-wave components which are absent in the quark model. This points towards the extraordinary nature of this state which still needs to be explored further.

A similar picture is observed in the various $\Delta$ channels. The quantitative agreement between the quark-diquark and the three-body approach is somewhat less pronounced than in the nucleon

\footnotetext{
${ }^{1}$ The mass evolution of the Roper with varying pion mass is discussed in [17] and compared with results from lattice QCD.
} 

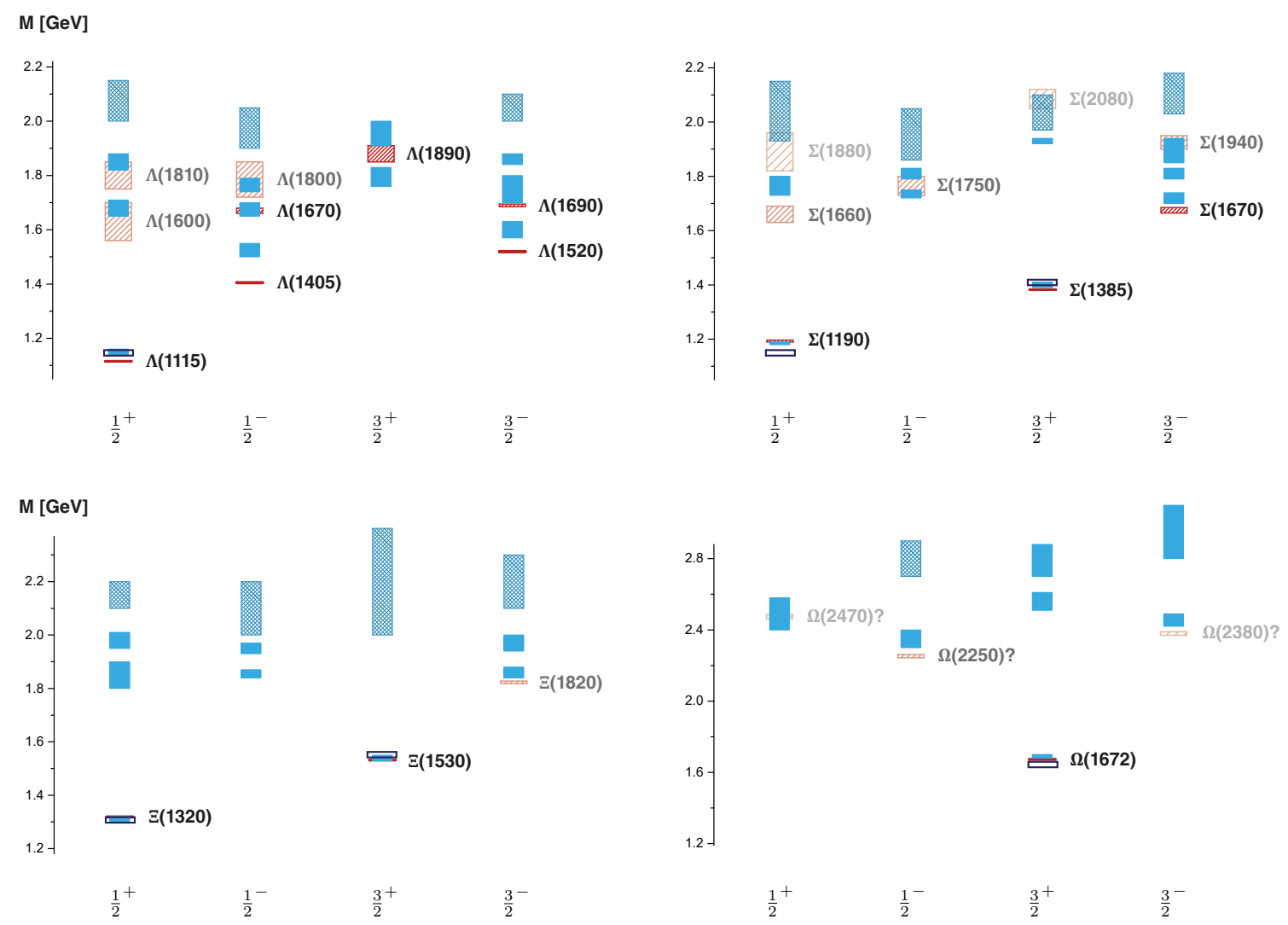

Figure 4: Single, double and triple strange baryon spectrum for $J^{P}=1 / 2^{ \pm}$and $3 / 2^{ \pm}$states determined within rainbow-ladder [24, 20] compared to PDG values [5]. Light-blue checkered boxes contain more than one state which are predicted in the same mass region but cannot yet be well enough resolved.

case but still satisfied on a semi-quantitative level. In general, not only the first radial excitations but also the second and third ones are close to experimental states. We therefore arrive at a consistent and quantitative description of the light baryon spectrum below $2 \mathrm{GeV}$.

Of course this does not mean that beyond rainbow-ladder effects, like pion-cloud and coupledchannel effects, or further non-Abelian corrections on the gluon level are absent [25]. First of all, effects that would reduce a baryon's mass and the pion decay constant by the same percentage trivially drop out in our plots since we set the scale by the experimental value of $f_{\pi}$. Thus, only the net effects remain visible. Our results then merely suggest that such net effects are opposite in sign and largely cancel each other in the nucleon and $\Delta(1232)$ channels, whereas in the other channels there is a net effect that can be largely absorbed by a (the third) parameter of the effective interaction. In fact, in the meson sector there are strong indications that cancellations between nonAbelian corrections [26] and pion cloud effects [27] occur. Both effects have already been explored for baryons on an exploratory level $[28,29]$ and work will continue in this direction.

Our results for the spectrum of single, double and triple strange baryons again from the threebody equation [24] as well as in the quark-diquark picture [20] are shown in Fig. 4. We use the same underlying quark-gluon interaction as for the light quarks assuming that a potential flavour dependence of the (dressed) quark-gluon interaction is mild, at least within flavour $S U(3)$. The re- 
sulting spectrum is again in overall good agreement with the experimental results, where available. In the single strange $\Lambda$ sector we obtain good agreement with the data for ground and excited states with $J=1 / 2^{ \pm}$with a general tendency of a slight overestimation of the masses in our approach. The exception is the $\Lambda(1405)$, which comes out much too heavy. This, however, ties in with the decades-long debate on this state indicating a composite structure beyond a three-quark description, see e.g. $[30,31,32,33,34]$ and references therein. In the channel with $J=3 / 2^{-}$the tendency of slight overestimation of the masses is repeated again and we predict additional excited states above the experimentally seen $\Lambda(1520)$ and $\Lambda(1690)$. The situation in the opposite parity channel with $J=3 / 2^{+}$is interesting, with the experimentally well-established $\Lambda(1890)$ sitting right in between the two states we find in this channel below $2 \mathrm{GeV}$. Certainly, this requires further studies on the theory as well as the experimental side. Note also that a similar situation is encountered in the quark model of Ref. [35].

In the single strange $\Sigma$ sector the experimental situation is less developed than for the $\Lambda$ baryons. Whereas the well-established ground states are well reproduced by our approach, the matching of the excited states needs further clarification, at least for the channels other than the $J=1 / 2^{+}$, where we find one-to-one agreement. On the other hand, the experimental analysis has made tremendous progress and is continually improving: An analysis of data on strangeness production by the Bonn-Gatchina group suggests three states in the $J=1 / 2^{-}$channel [36] with masses in agreement with our prediction (and the one of the quark model [35]). Similarly interesting may be an analysis in the $J=3 / 2^{-}$channel, where our calculation predicts at least four states below 2.2 $\mathrm{GeV}$ in contrast to three states in the quark model [35] with only two states in the PDG so far.

Finally, we enter uncharted territory in the double and triple strange baryon sector. Our results for the ground states are in good agreement with the experimentally established ones; all excited states and even the quantum numbers and masses of some ground states are predictions which can be explored in current and future experiments such as LHCb and PANDA/FAIR. Also very encouraging are the results for the strange baryon sector reported for the three-body framework in [24]. Here the masses of ground-state octet and decuplet baryons have been reproduced on the few-percent level, while the calculation of excited states still needs to be performed.

\section{Four-quark states}

Solving the Bethe-Salpeter and Faddeev equations in the Dyson-Schwinger framework is by now an endeavour that is technically well established and under control, see [37] for a summary on the technical aspects. In contrast, the technical challenges of the four-body equation are far more advanced due to the proliferation of tensor structures and independent momenta. All results so far must therefore be considered exploratory $[38,39,40]$. Nevertheless, a number of interesting points can be made, in particular with respect to the possibility to treat not only bound states but also resonances in this framework.

To this end we briefly need to introduce some conventions concerning total and relative momenta. Consider the four-quark Faddeev-Yakubowsky equation displayed in Fig. 5, where we have omitted diagrams with irreducible three- and four-body forces simply due to lack of ability to treat these numerically. From the four momenta of the individual (anti-)quarks we can form three inde- 


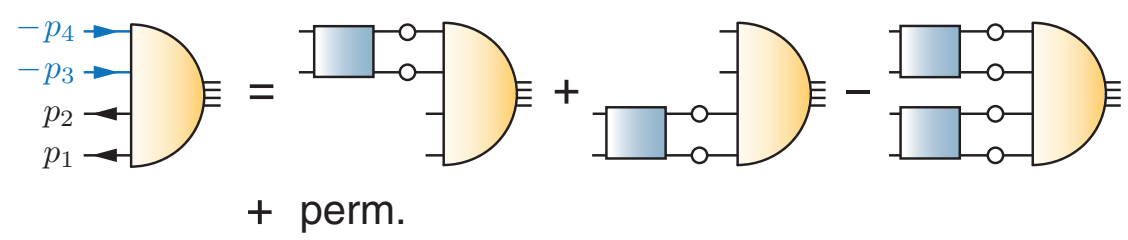

Figure 5: Four-quark Faddeev-Yakubowsky equation.

pendent linear combinations:

$$
\begin{aligned}
& p=\frac{1}{2}\left(p_{2}+p_{3}-p_{1}-p_{4}\right), \\
& q=\frac{1}{2}\left(p_{3}+p_{1}-p_{2}-p_{4}\right), \quad P=\sum_{i=1}^{4} p_{i} . \\
& k=\frac{1}{2}\left(p_{1}+p_{2}-p_{3}-p_{4}\right),
\end{aligned}
$$

Each of these contains total momenta of different internal two-body clusters. For example, the momentum $p$ contains the total momenta of two quark-antiquark states where the (anti-)quarks 2 and 3 as well as 1 and 4 are combined. The momentum $q$ contains the total momenta of the other possible combination of two quark-antiquark states, whereas the momentum $k$ describes the total momenta of an internal diquark and an internal antidiquark in the four-quark state. These clusters are interesting since they may go on-shell provided that the sum of the masses of the clusters are smaller than the mass of the four-body state. If this occurs in color-singlet channels, the four-quark state becomes a resonance in the two-body hadronic system of the corresponding clusters.

A prime example of this possibility occurs in the light quark sector: the lightest scalar meson nonet has long been advocated to correspond not to quark-antiquark systems but to ones with fourquark content [41, 42]. The resulting mass ordering is then (and only then) in agreement with the experimentally observed spectrum. Indeed, the lightest state in this nonet, the $f_{0}(500)$, is reconstructed in pion scattering events $[43,44]$ and thus a resonance in the $\pi \pi$ system. Translated into our four-body description, we expect strong and resonant contributions to the four-body equation from contributions in the momenta $p$ or $q$ which allow for the dynamical clustering of (anti-)quarks forming pions.

In order to allow for this to happen, two conditions have to be satisfied: (i) The two-body interaction has to support chiral symmetry breaking is such a way that pions as (pseudo-)Goldstone bosons may emerge. This is indeed the case for the rainbow-ladder interaction used in the previous chapter (as discussed and celebrated in many publications, see e.g. [45] for an early review), which consequently has also been employed in the four-body equation [40]. (ii) The technical treatment of the equation has to be sufficiently rich such that the momentum dependencies in question are taken into account. In this respect an ordering scheme according to the permutation group $S_{4}$ classified in [39] is extremely helpful. It allows one to consider linear combinations of the Lorentzinvariant variables $p^{2}, q^{2}, k^{2}, p \cdot q, p \cdot P$, etc. such that they form multiplets of the permutation group. Without going into technical details (discussed in $[39,40]$ ) it is sufficient to note that there is a singlet which mainly plays the role of setting the scale of the problem, a doublet containing all possibilities of resonances in sub-clusters as described above, and two triplets which turn out to be not tremendously important. 

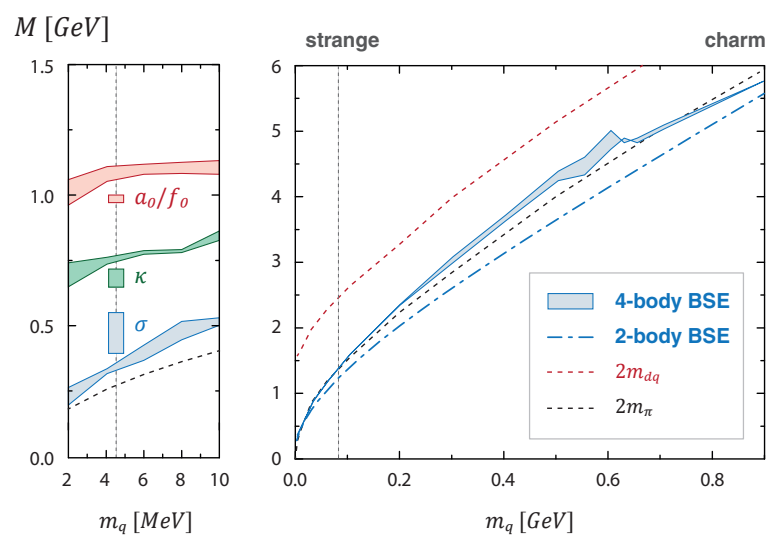

Figure 6: Left panel: tetraquark masses at fixed strange quark mass and varying light quark mass. The bands contain the extrapolation errors and the dotted curve is the two-pion threshold. The vertical dotted line shows the physical $u / d$ mass and the vertical bars are the experimental values for the masses [5]. Right panel: quark-mass dependence of the tetraquark mass with four identical quarks. The band is the present result from the four-body equation with extrapolation errors, compared to that obtained with coupled mesonmeson/ $d q-\overline{d q}$ equations (dash-dotted curve) [38]. The dotted curves are the respective thresholds.

Keeping in mind that four internal up/down quark propagators each describe a (anti-)quark with a mass of roughly $350 \mathrm{MeV}$ in the low momentum limit (see e.g. [1]), it is interesting to follow the evolution of the resulting four-body mass with scalar quantum numbers $J^{P C}=0^{++}$ using different levels of approximations in its the wave function: If only the singlet variable in the momenta is taken into account, or the singlet variable is combined with triplets, one finds masses of the order of $1.2 \ldots 1.5 \mathrm{GeV}$. These are typical for bound states of four constituents with the masses of the quarks. If, however, the wave functions are also allowed to depend on the momentum variables sitting in the doublet, the picture changes completely and the resulting mass of the fourbody state is of the order of $350 \ldots 400 \mathrm{MeV}$. This is the mass region expected for a four-body state that is a resonance in the $\pi \pi$-system - the $f_{0}(500)$. Indeed, close inspection of the equation reveals that the two-body clusters with pseudoscalar quantum numbers dominate the calculation.

This consideration can be extended to include strange quarks such that masses for the $\kappa$ and the $a_{0} / f_{0}$ mesons can be extracted. These are shown in the bands on the left panel of Fig. 6 together with the experimental values of the masses [5]. The qualitative agreement is very satisfactory considering the exploratory nature of the calculation [40]. In the right panel we show the transition from a resonance with mass larger than $2 m_{\pi}$ to a bound state, when the bare mass of the up/down quark is increased. This transition happens slightly below the charm quark region, such that the four-charm tetraquark is a bound state (at this level of approximation). Also shown are results from a two-body version of the Faddeev-Yakubowsky equation in analogy to the diquark approximation of the baryon, see [38] for details.

The main point to learn from this exploratory calculation is that it is possible in principle to describe resonances in the DSE/BSE framework and that the equation itself teaches us which internal clusters are the most important ones. That the scalar four-quark state is dominated by a configuration with the quantum numbers of two pions is not an input, but a result of the calculation. 


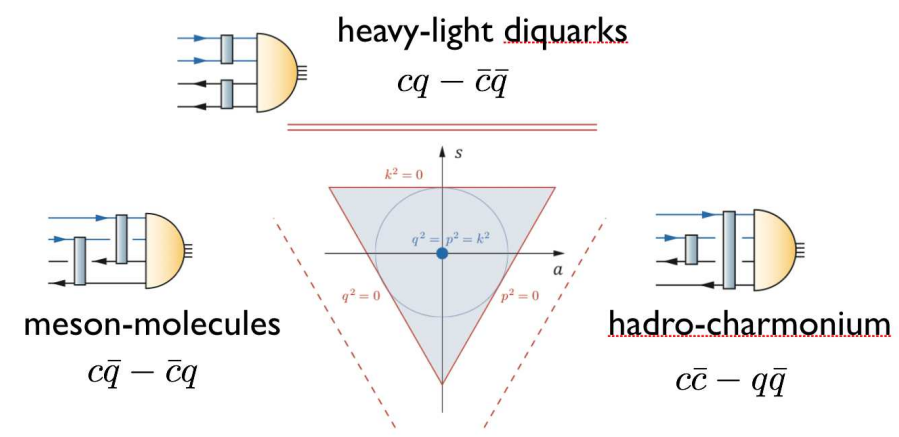

Figure 7: Kinematical situation for heavy-light four-(anti-)quark states in the Faddeev-Yakubowsky equations. The variables $s$ and $a$ define the doublet (see text), whereas the directions of $p, q$ and $k$ are indicated by the labels $p^{2}=0$ etc. which define the border of the shaded integration region inside the FaddeevYakubowsky equation.

This becomes even more interesting when combinations of heavy and light quarks are considered. Much of the present discussion of potential exotic states in the charm quark energy region revolves around the question whether candidates for exotic four-quark states are better described by a meson molecule picture, a tetraquark picture in terms of diquarks and anti-diquarks, or a hadrocharmonium picture with two heavy (anti-)quarks sitting in the center of a system where they are enveloped by a light quark-antiquark pair. In principle this can be decided by the kinematics of the four-quark system. In Fig. 7 we display the kinematic situation in the plane spanned by the two doublet momentum variables $a$ and $s$,

$$
a=\sqrt{3} \frac{q^{2}-p^{2}}{4 S_{0}}, \quad s=\frac{p^{2}+q^{2}-2 k^{2}}{4 S_{0}},
$$

with $S_{0}=\left(p^{2}+q^{2}+k^{2}\right) / 4$. The shaded region is the integration region of the four-body equation. For bound states, the kinematical situation where one of the internal clusters can get on-shell, displayed by the dashed and double lines, are outside the integration region. If one of these clusters comes close to the boundary of the integration region, then the corresponding states start to influence the four-body calculation; for resonances such as the light meson nonet discussed above, the cluster poles actually move inside the integration region and dominate everything. Of course, whether a given cluster pole does or does not influence the calculation depends on the masses of the clustered objects. For a state such as the $X(3872)$, which has a mass very close (or identical) to the $D^{0} \bar{D}^{0 *}$ threshold, the corresponding clusters in the meson-molecule direction sit right on the edge of the integration region, which definitely will have a strong impact. Whether the resulting state (and other candidates for exotics) can be described well in the present framework is a highly non-trivial, technically very difficult and therefore open question that is interesting to be further explored in the future.

\section{Conclusions}

Hadron spectroscopy remains a very active and lively field. Dedicated experiments at many facilities such as Jefferson Lab, BESIII, ELSA, J-PARC, LHCb, MAMI and the future PANDA/FAIR 
experiment are contributing and will continue to contribute to our understanding of the non-exotic and exotic hadronic sectors. On the theory side, the past years have seen continuous advances in connecting the underlying quark and gluon interactions of QCD with baryon phenomenology.

The challenges in functional approaches are to improve the approximation schemes beyond simple modelling. However, much can be achieved in the way of understanding the underlying mechanisms that generate the observed phenomena without the need to enter the realm of high precision physics on the sub-percent level. Indeed, using a well-established rainbow-ladder approximation we found a spectrum of ground and excited states of light and strange baryons in mostly one-to-one agreement with experiment. This highly non-trivial result is very encouraging and suggests further extensions of our work to quantum numbers beyond $J=1 / 2$ and $J=3 / 2$. Predictions for ground and excited states in the strange quark sector may be tested in the future.

On the other hand, we have seen that exploratory calculations in the four-quark sector promise the possibility to explore bound states and resonances in the light but also the heavy-light and heavy quark sector. Provided that the multitude of technical difficulties associated with these calculations can be overcome, this seems to be a very promising line of research in the future with a lot of potential for creating a complementarity tool to effective theories and lattice QCD for the interpretation of these states.

\section{Acknowledgements}

The work reported here would not have been possible without the support from the Deutsche Forschungsgemeinschaft within the SFB/TR16, the research grant FI970/11-1, and the FCT Investigator Grant IF/00898/2015. We furthermore acknowledge support from the Helmholtz International Center for FAIR within the LOEWE program of the State of Hesse.

\section{References}

[1] G. Eichmann, H. Sanchis-Alepuz, R. Williams, R. Alkofer and C. S. Fischer, Prog. Part. Nucl. Phys. 91 (2016) 1-100, [1606.09602].

[2] A. V. Sarantsev et al., Phys. Lett. B659 (2008) 94-100, [0 707.3591$].$

[3] I. G. Aznauryan et al., Phys. Rev. C80 (2009) 055203, [0 909.2349 ].

[4] I. G. Aznauryan et al., Int. J. Mod. Phys. E22 (2013) 1330015, [1212 . 4891$].$

[5] C. Patrignani et al., Chin. Phys. C40 (2016) 100001.

[6] C. A. Meyer and E. S. Swanson, Prog. Part. Nucl. Phys. 82 (2015) 21-58, [1502.07276].

[7] H.-X. Chen, W. Chen, X. Liu and S.-L. Zhu, Phys. Rept. 639 (2016) 1-121, [1601. 02092 ].

[8] A. Esposito, A. Pilloni and A. D. Polosa, Phys. Rept. 668 (2016) 1-97, [1611.07920].

[9] S. Capstick and W. Roberts, Prog. Part. Nucl. Phys. 45 (2000) S241-S331, [nucl-th/ 0008028 ].

[10] E. Klempt and J.-M. Richard, Rev. Mod. Phys. 82 (2010) 1095-1153, [0 901.2055$].$

[11] P. Maris, Few Body Syst. 32 (2002) 41-52, [nucl-th/0204020].

[12] G. Eichmann, Few Body Syst. 57 (2016) 965-973, [1602.03462].

[13] M. Oettel, G. Hellstern, R. Alkofer and H. Reinhardt, Phys. Rev. C58 (1998) 2459-2477, [nucl-th/9805054]. 
[14] M. Oettel, R. Alkofer and L. von Smekal, Eur. Phys. J. A8 (2000) 553-566, [nucl-th/ 0006082 ].

[15] J. Segovia, B. El-Bennich, E. Rojas, I. C. Cloet, C. D. Roberts, S.-S. Xu et al., Phys. Rev. Lett. 115 (2015) 171801, [1504.04386].

[16] P. Maris and P. C. Tandy, Phys. Rev. C60 (1999) 055214, [nucl-th/9905056].

[17] G. Eichmann, C. S. Fischer and H. Sanchis-Alepuz, Phys. Rev. D94 (2016) 094033, [1607 . 05748 ].

[18] A. Krassnigg, Phys. Rev. D80 (2009) 114010, [0909. 4016 ].

[19] H. L. L. Roberts, L. Chang, I. C. Cloet and C. D. Roberts, Few Body Syst. 51 (2011) 1-25, [1101.4244].

[20] G. Eichmann and C. S. Fischer, in preparation .

[21] G. Eichmann, R. Alkofer, A. Krassnigg and D. Nicmorus, Phys. Rev. Lett. 104 (2010) 201601, [0912.2246].

[22] H. Sanchis-Alepuz, G. Eichmann, S. Villalba-Chavez and R. Alkofer, Phys. Rev. D84 (2011) 096003, [1109.0199].

[23] C. Chen, B. El-Bennich, C. D. Roberts, S. M. Schmidt, J. Segovia and S. Wan, 1711.03142.

[24] H. Sanchis-Alepuz and C. S. Fischer, Phys. Rev. D90 (2014) 096001, [1 408 . 5577 ].

[25] R. Williams, C. S. Fischer and W. Heupel, Phys. Rev. D93 (2016) 034026, [1512. 00455 ].

[26] C. S. Fischer and R. Williams, Phys. Rev. Lett. 103 (2009) 122001, [0 905 . 2291].

[27] C. S. Fischer and R. Williams, Phys. Rev. D78 (2008) 074006, [0 808 . 3372].

[28] H. Sanchis-Alepuz, C. S. Fischer and S. Kubrak, Phys. Lett. B733 (2014) 151-157, [1401. 3183 ].

[29] H. Sanchis-Alepuz and R. Williams, Phys. Lett. B749 (2015) 592-596, [1504 . 07776 ].

[30] R. H. Dalitz, T. C. Wong and G. Rajasekaran, Phys. Rev. 153 (1967) 1617-1623.

[31] E. A. Veit, B. K. Jennings, A. W. Thomas and R. C. Barrett, Phys. Rev. D31 (1985) 1033.

[32] B. J. Menadue, W. Kamleh, D. B. Leinweber and M. S. Mahbub, Phys. Rev. Lett. 108 (2012) 112001, [1109.6716].

[33] M. Mai and U.-G. Meissner, Nucl. Phys. A900 (2013) 51 - 64, [1202 . 2030].

[34] R. Molina and M. Döring, Phys. Rev. D94 (2016) 056010, [1512. 05831 ].

[35] U. Loring, B. C. Metsch and H. R. Petry, Eur. Phys. J. A10 (2001) 447-486, [hep-ph/ 0103290 ].

[36] A. V. Sarantsev et al., these proceedings .

[37] H. Sanchis-Alepuz and R. Williams, 1710.04903.

[38] W. Heupel, G. Eichmann and C. S. Fischer, Phys. Lett. B718 (2012) 545-549, [1206. 5129 ].

[39] G. Eichmann, C. S. Fischer and W. Heupel, Phys. Rev. D92 (2015) 056006, [1505. 06336 ].

[40] G. Eichmann, C. S. Fischer and W. Heupel, Phys. Lett. B753 (2016) 282-287, [1508 . 07178].

[41] R. L. Jaffe, Phys. Rev. D15 (1977) 267.

[42] J. R. Pelaez, Phys. Rept. 658 (2016) 1, [1510.00653].

[43] I. Caprini, G. Colangelo and H. Leutwyler, Phys. Rev. Lett. 96 (2006) 132001, [hep-ph/ 0512364$].$

[44] R. Garcia-Martin, R. Kaminski, J. R. Pelaez and J. Ruiz de Elvira, Phys. Rev. Lett. 107 (2011) 072001, [1107.1635].

[45] P. Maris and C. D. Roberts, Int. J. Mod. Phys. E12 (2003) 297-365, [nucl-th/ 0301049 ]. 